\title{
Influence d'une réhydratation préalable sur la germination in vitro du pollen de douglas (Pseudotsuga menziesii) après conservation
}

\author{
J.P. CHARPENTIER 11 et M. BONNET-MASIMBERT \\ I.N.R.A., Station d'A mélioration des Arbres forestiers, \\ Centre de Recherches d'Orléans, Ardon, F 45160 Olivet
}

\begin{abstract}
Résumé
Après avoir précisé les conditions de germination in vitro du pollen de douglas (Pseudotsuga menziesii), nous avons défini les modalités d'utilisation d'un pollen déshydraté avant conservation. Pour la mise en route du processus de germination. une phase préalable d'hydratation ménagée s'est avérée indispensable. Cette hydratation s'effectue bien à $26^{\circ} \mathrm{C}$ pendant 16 h., en atmosphère saturée d'eau. Elle permet à certains lots de passer de 13 p. 100 à 65 p. 100 de germination. En parallèle, une étude cytologique a révélé, au niveau ultrastructural, une évolution du cytoplasme au cours de l'hydratation. Celle-ci concerne notamment le chondriome et l'appareil vacuolairc.
\end{abstract}

\section{Introduction}

Toutes les techniques de conservation du pollen, revues notamment par SNYder \& Cl.ausen (1974), reposent sur l'utilisation de températures basses nécessitant une déshydratation parfois très importante du pollen. Or, si de nombreuses études concernent les techniques de conservation elles-mêmes, bien peu šintéressent à la manière dutiliser le pollen après conservation. Cependant certains auteurs signalent qu'une exposition en atmosphère humide, le plus souvent réalisée en conditions non contrôlées, favorise la germination. C"est le cas de Duffilid \& SNow (1941) sur Pinus strobus et P. resinosa, VISSER (1955) sur poirier et pommier, KING (1965) sur plusieurs angiospermes, JenseN (1970) sur quelques gymnospermes, MURlen et al. (1979) sur douglas. Simons ot al. (1972) sur Lilium longifolitim, Bar-Shalom \& MatTsSon (1976) sur divers angiospermes et Gilıssen (1977) sur Petumia hybrida considèrent cette phase de réhydratation comme essentielle pour l'obtention d'une honne germination, et notent l'importance du mode d'hydratation.

(1) Adresse présente : Laboratoire d’Etude des Composés Phénoliques, U.E.R. SciencesOrléans, F 45046 Orléans Cedex. 
Un travail effectué dans notre Station depuis 1979 sur la conservation et la germination du pollen de douglas (Pseudotsuga menziesii Mirb. Franco) nous a conduits à supposer que de mauvais taux de germination pouvaient parfois n'être dus qu'à des difficultés d'hydratation. Cette étude visait donc, après mise au point d'une méthode de germination, à préciser, y compris sous l'angle cytologique, la nature et l'ampleur du phénomène et à définir les modalités permettant de le maîtriser.

\section{Matériel et techniques}

\subsection{Pollen}

Les essais préliminaires et mises au point de techniques ont été effectués avec du pollen récolté en 1979 et en 1980 et conservé à des températures variées allant de $+4^{\circ} \mathrm{C}$ à $-17^{\circ} \mathrm{C}$. Les essais précis concernant la déshydratation et la réhydratation portent sur du pollen frais récolté au printemps 1981. Dans ces essais, la déshydratation, en vue d'obtenir une gamme de teneurs en eau de 3,5 p. 100 à 8,5 p. 100 est réalisée en plaçant le pollen, en fine couche, sur papier filtre déposé à la surface d'un lit d'actigel (sel déshydratant), à l'intérieur d'une enceinte hermétique. Le pollen est ensuite placé, en flacons étanches, à $-1{ }^{\circ} \mathrm{C}$.

\subsection{Test de germination}

Il convient tout d'abord de rappeler que chez le douglas, il n'y a individualisation d'un tube pollinique, d'ailleurs très court, qu'au contact du nucelle (BARNER \& ChristianSEN, 1962 ; Allen \& OWens, 1972, etc.). Par contre, c'est l'ensemble du grain qui s'allonge pour former une structure tubulaire. Nous avons donc considéré, par extension, que ce processus d'élongation caractérisait un pollen en cours de germination.

Les tests ont été réalisés en germoirs, à $25 \pm 1$ "C, à l'obscurité, en ambiance saturée d'humidité, selon la technique dite en «goutte pendante». Le milieu de base pour la germination est celui de BREWBAKER \& KWACK (1963) $\left(\mathrm{H}_{3} \mathrm{BO}_{3}(100 \mathrm{mg} / \mathrm{l})\right.$; $\mathrm{Ca}\left(\mathrm{NO}_{3}\right)_{2}, 4 \mathrm{H}_{2} \mathrm{O}(300 \mathrm{mg} / \mathrm{l}) ; \mathrm{KNO}_{3}(100 \mathrm{mg} / \mathrm{l}) ; \mathrm{Mg} \mathrm{SO}_{4}, 7 \mathrm{H}_{2} \mathrm{O}(200 \mathrm{mg} / \mathrm{l})$. Il est stérilisé par autoclavage à $120^{\circ} \mathrm{C}$ pendant 20 minutes. L'étude de l'influence du pH du milieu a révélé (tabl. 1) que c'était entre 4,3 et 6,1 (pH obtenu avec de l'eau distillée, sans ajustement) que les taux de germination étaient les meilleurs. La comparaison de milieux avec ou sans saccharose $(5$ p. 100) s'est avérée défavorable au milieu sucré, qui stimule surtout le développement des infections et provoque un éclatement des grains de pollen, sans stimulation notable de leur croissance. Les tests de germination ont donc été réalisés en l'absence de sucre. Par contre, nous avons ajouté dans le milieu, après autoclavage, $15 \mu \mathrm{g} / \mathrm{ml}$ de nystatine et $15 \mu \mathrm{g} / \mathrm{ml}$ de chloramphénicol (Muren et al., 1979), qui, après comparaison avec d'autres produits phytosanitaires se sont avérés les plus efficaces pour empêcher la prolifération des bactéries et champignons.

\subsection{Réhydratation}

Les échantillons de pollen, placés en boîtes de Pétri ouvertes, sont mis dans un bocal étanche au-dessus d'une couche d'eau distillée (hygrométrie saturante) ou de l'une des quatre solutions saturées suivantes, permettant d'établir dans le bocal différents 


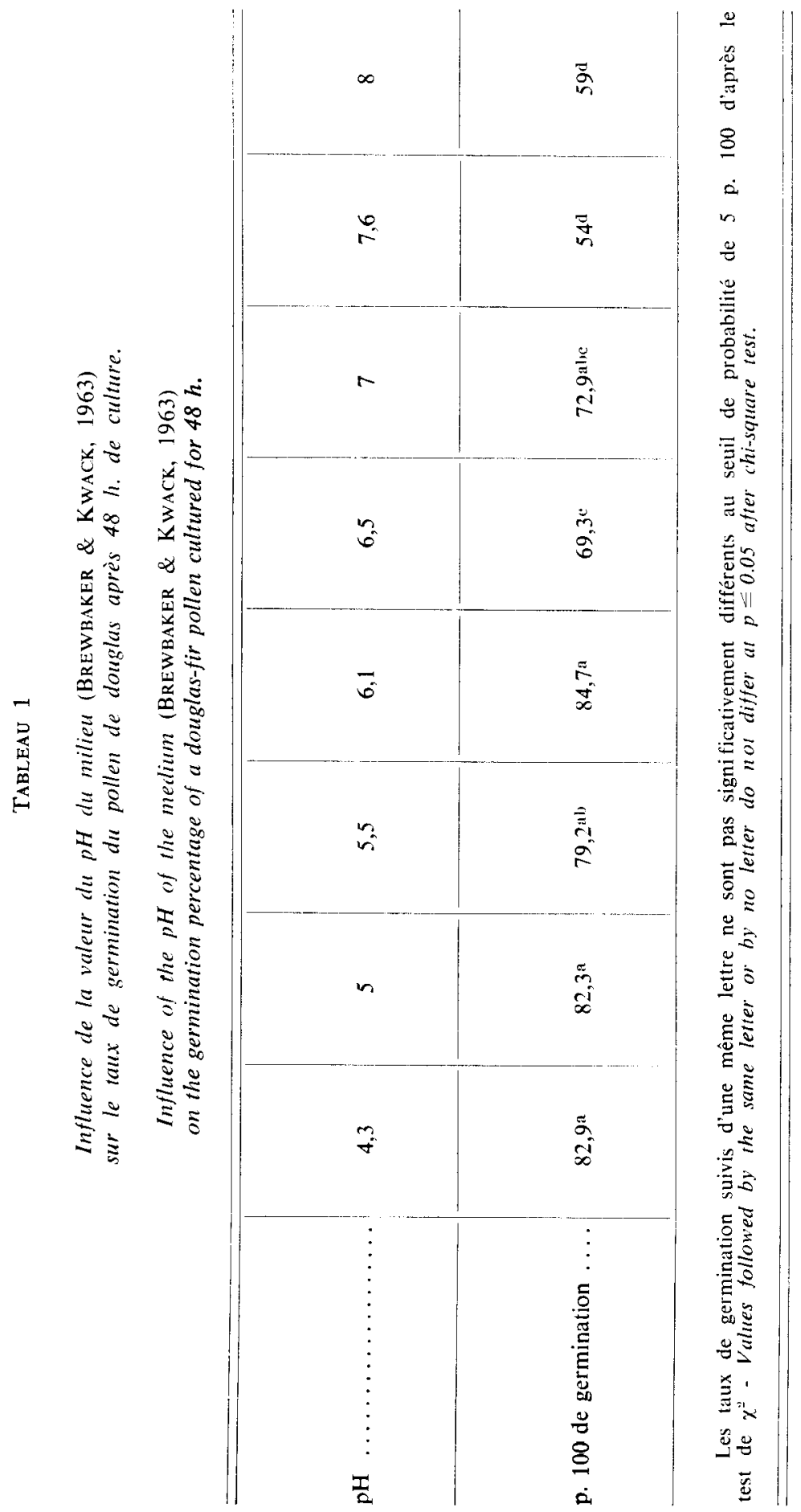


degrés d'humidité relative $(\mathrm{HR})$ (valeurs données à 20 " $\mathrm{C}$ ): chlorure de calcium $(\mathrm{HR}=$ 35 p. 100), nitrate de calcium $(H R=55$ p. 100), nitrate dammonium $(H R=63$ p. 100), sulfate d’ammonium $(H R=80$ p. 100). La température avait été définie dans un essai préliminaire, après $18 \mathrm{~h}$ d'hydratation (en hygrométrie saturante), à l'une des trois températures suivantes : $+4{ }^{\circ} \mathrm{C},+20{ }^{\prime \prime} \mathrm{C}$ et $+26^{\circ} \mathrm{C}$. Contrairement à Muren et al. (1979) qui utilisaient +4 "C, nous avons obtenu les meilleurs résultats à 26 "C. C'est donc cette température qui a été retenue pour les réhydratations. Après cette phase, une partie du pollen est mise en germination, le reste servant à la mesure de la teneur en eau. Celle-ci est calculée par rapport au poids frais, sur 100 à $200 \mathrm{mg}$ de pollen, par mesure de la perte en eau après une heure de séchage à 105 " $\mathrm{C}$.

\subsection{Microscopie électronique}

Le pollen est daabord fixé par une solution de glutaraldéhyde à 4 p. 100 dans un tampon cacodylate de sodium à pH 7. Il est ensuite post-fixé par une solution de tétroxyde d'osmium à 1 p. 100 dans un tampon véronal à $\mathrm{pH} \mathrm{7,4.} \mathrm{Après} \mathrm{chaque} \mathrm{bain,}$ le pollen est récupéré par une légère centrifugation $(10 \mathrm{mn}$ à $1000 \mathrm{t} / \mathrm{mn})$. Le culot de pollen est ensuite inclus dans la gélose à $10 \mathrm{~g} / 1$. Puis des morceaux de gélose contenant une forte densité de pollen sont découpés et inclus dans l'araldite selon la technique de Glauert \& Glauert (1958). Les coupes ultrafines débitées à l'ultra microtome peuvent être observées sans coloration, ou bien contrastées, soit par le permanganate de potassium à 1 p. 100 dans l'eau, soit par l'acétate d'uranyle et le citrate de plomb. Les observations ont été faites à l'aide d'un microscope électronique Hitachi HU 1 E.

\section{Résultats et commentaires}

\subsection{Influence de la rehydratation sur le taux de germination}

Un premier essai a porté sur deux lots de polten conservés pendant 3 mois en récipients étanches, à -1 " $\mathrm{C}$, avec des teneurs en eau respectivement de 5,2 et 7,9 p. 100. Nous avons étudié l'influence, sur la teneur en eau et la germination, de l'humidité relative de latmosphère de réhydratation, pour une même duréc de $18 \mathrm{~h}$. Les résultats obtenus, après $48 \mathrm{~h}$. de germination, sont donnés dans le tableau 2. Les deux lots manifestent une même évolution : augmentation du taux de germination avec l'augmentation de teneur en eau. Cette relation est beaucoup plus prononcée pour le lot 1 qui passe par hydratation à $H R=100$ p. 100 d'un taux de germination de 4,3 à 54,5 p. 100 . Le lot 2, moins déshydraté au départ, gagne 20 p. 100 de germination (différence significative) grâce à la phase dhydratation. Enfin, il est à noter que, à teneur équivalente, les taux de germination du lot 1 restent toujours nettement inférieurs à ceux du lot 2, phénomène qui ne s'atténue qua à partir de 22 p. 100 de teneur en eau. Il est probable que ceci traduit des difficultés de mise en route des processus germinatifs lorsque la déshydratation préalable a été trop importante. Enfin, dans les deux cas, c'est à $\mathrm{HR}=100$ p. 100 que la meilleure germination est obtenue.

Nous avons alors, dans un deuxième essai, étudié linfluence de la durée de la phase d'hydratation sur la teneur en eau et la germination (après 30 h.). L'essai a été conciuit à $H R=100$ p. 100 . Les résultats sont donnés dans le tableau 3. Là aussi le taux de germination seélève rapidement avec les teneurs en eau croissantes. Déjà, après 
1 heure, le taux est passé de 13,2 à 41,9 p. 100 . Il passera à 65,8 p. 100 après 16 h. Des durées plus longues n'ont pas été expérimentées, mais it apparaît déjà possible de stimuler considérablement la germination in vitro par simple application d'une phase de réhydratation préalable.

\section{TABLenU 2}

Influence de l'humidité relative (HR) de l'atmosphère dans laquelle s'effectue la réhydratation $(18 \mathrm{~h})$ sur la teneur en eau et le taux de germination (après 48 h) de deux lots de pollen de douglas à teneur en eau initiale différente.

Relative humidity of the atmosphere for the $18 \mathrm{~h}$ rehydration phase: its influence on the moisture content and on the germination percentage (after a $48 \mathrm{~h}$ culture) of two douglas-fir pollen lots, differing in their initial moisture content.

\begin{tabular}{|c|c|c|c|c|}
\hline \multirow[b]{2}{*}{$\begin{array}{l}\text { Humidité relative } \\
\text { de l'atmosphère }\end{array}$} & \multicolumn{2}{|c|}{ Lot 1} & \multicolumn{2}{|c|}{ Lot 2} \\
\hline & Teneur en eau & $\begin{array}{l}\text { p. } 100 \\
\text { germination } \\
\text { a } 48 \mathrm{~h}\end{array}$ & Teneur en eau & $\begin{array}{l}\text { p. } 100 \\
\text { germination } \\
\text { à } 48 \mathrm{~h}\end{array}$ \\
\hline Témoin non réhydraté & 5,2 & $4,3^{a}$ & 7,9 & $42,4^{\mathrm{a}}$ \\
\hline 35 p. 100 & 7,2 & $17,9^{\mathrm{b}}$ & 9,5 & 43 a \\
\hline 55 p. 100 & 13,2 & $24,1^{\text {lie }}$ & 14,6 & $54,8^{b}$ \\
\hline 63 p. 100 & 16 & $27,5^{\circ}$ & 15,5 & $56,2^{\text {be }}$ \\
\hline 80 p. 100 & 22 & $46,9 d$ & 21,5 & $59,9 \mathrm{bc}$ \\
\hline 100 p. 100 & 41,1 & 54,50 & 48 & $62,4^{c}$ \\
\hline
\end{tabular}

Dans une même colonne, les taux de germination suivis d'une même lettre ne sont pas significativement différents au seuil de probabilité de 5 p. 100 d'après le test de $\chi^{2}$ - Within each column, values followed by the same letler or by no letter do not differ at $p \leq 0.05$ after chi-square test.

\section{Tableau 3}

Influence de la durée de réhydratation $(H R=100 \mathrm{p} .100)$ sur la teneur en eau et le taux de germination d'un lot de pollen de douglas.

Influence of the length of the rehydration phase $(R H$ of the atmosphere $=100 \mathrm{p.} 100)$ on moisture content and germination percentage of one douglas-fir pollen lot.

\begin{tabular}{|c|c|c|c|c|c|c|c|c|c|}
\hline \multirow{2}{*}{ Temps d'hydratation } & \multicolumn{5}{|c|}{ Minutes } & \multicolumn{4}{|c|}{ Heures } \\
\hline & 0 & 5 & 10 & 15 & 30 & 1 & 3 & 5 & 16 \\
\hline Teneur en eau...... & 9,2 & 11,4 & 15,2 & 17,7 & 19,6 & $2 \cdot 1,2$ & 24,6 & 34,3 & 48,6 \\
\hline $\begin{array}{l}\text { Taux de germination à } \\
30 \mathrm{~h} \ldots \ldots \ldots \ldots \ldots\end{array}$ & $\begin{array}{c}13,2 \\
a\end{array}$ & $\begin{array}{c}25,3 \\
\mathrm{~b}\end{array}$ & $\begin{array}{c}27,8 \\
\text { be }\end{array}$ & $\begin{array}{c}29,3 \\
\text { be }\end{array}$ & $\begin{array}{c}32,8 \\
c\end{array}$ & $\begin{array}{c}41,9 \\
\mathrm{~d}\end{array}$ & $\begin{array}{c}44,4 \\
\text { de }\end{array}$ & $\begin{array}{r}50 \\
\mathrm{e}\end{array}$ & $\underset{\mathrm{f}}{65,8}$ \\
\hline
\end{tabular}

Les taux de germination sous lesquels se trouvent des lettres identiques ne sont pas significativement différents au seuil de probabilité de 5 p. 100 d'après le test de $\chi^{2}$ - Values followed by the same letter or by no letter do not differ at $p=0.05$ after chi-square test. 

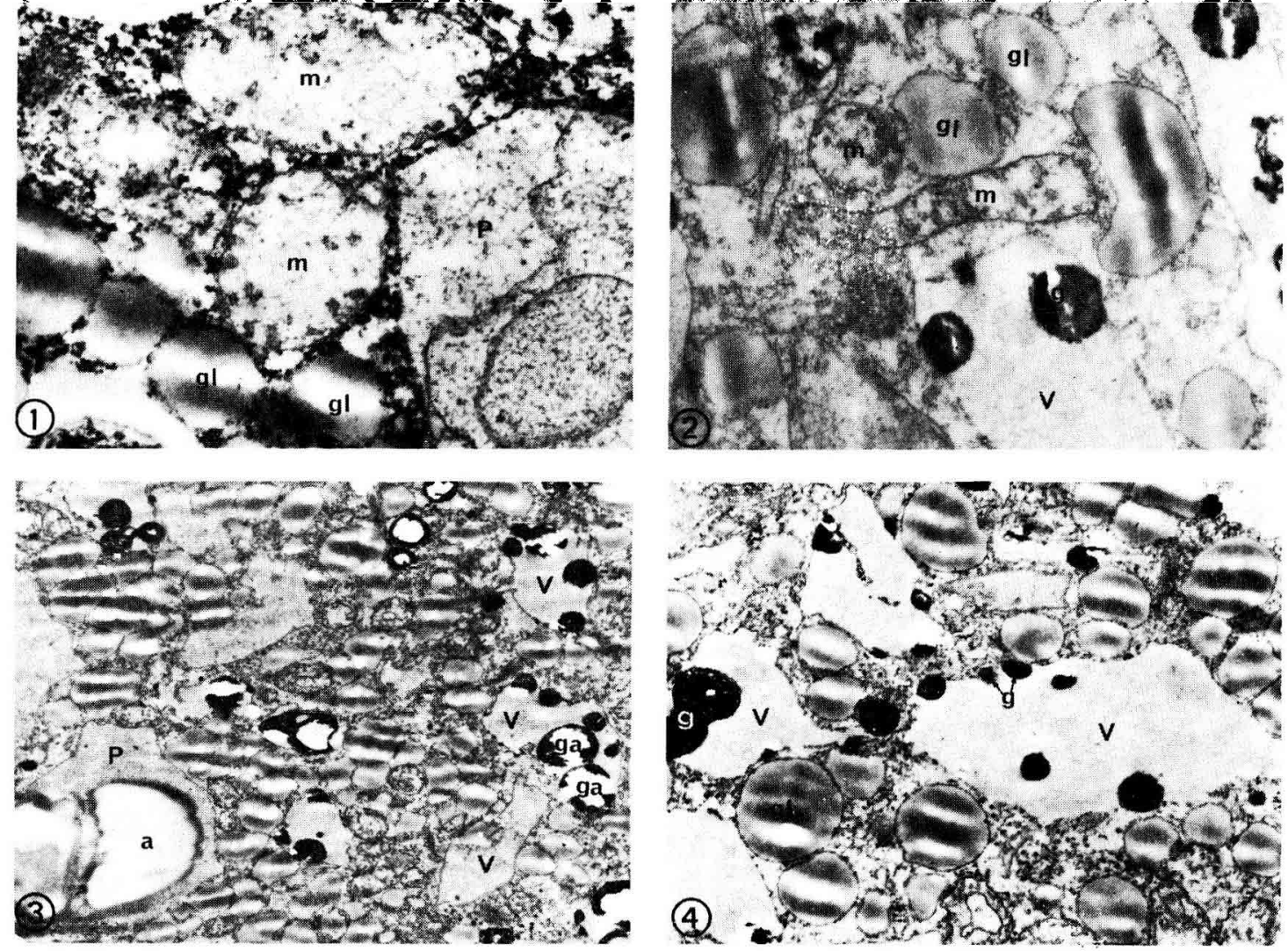

Cytologie de grains de pollen de douglas à différents degrés d'hydratation.

The cytology of some pollen grains of douglas-fir at different moisture content.

Abréviations utilisées : $m$, mitochondrie; $p$, plaste; $a$, grain d'amidon; $v$, vacuole; gl, globule lipidique; ga, grain d'aleurone; g, globoide.

Abbrevations used : $m$, mitochondrion; $p$, plastid; $a$, starch grain; $v$, vacuole; gl, lipid bodies; ga, aleuron grain; g, globoid.

FIG. 1

Coloration au plomb $(\times 12000)$. Cytoplasme de la cellule reproductrice d'un grain peu hydraté : mitochondries claires, arrondies, avec peu de crêtes.

Nombreux globules lipidiques.

Lead staining $(\times 12,000)$. Cytoplasm of the generative cell of a pollen grain with low hydration : mitochondria are clear, rounded with few crest. Many lipid bodies.

FIG. 2

Coloration $\mathrm{KMnO}_{4}(\times 9400)$. Cytoplasme d'un grain réhydraté.

Les vacuoles sont bien développées, les mitochondries s'allongent, avec formation de crêtes.

$\mathrm{KMnO}_{4}$ staining $(\times 9,400)$. Cytoplasm of a rehydrated grain.

Vacuoles are well developped. Mitochondria are lengthenning, with formation of crests.

FIG. 3

Coloration au plomb $(\times 3600)$. Grain en cours de réhydratation.

Développement progressif des vacuoles à partir de grains d'aleurone.

Présence de globoides rejetés contre le tonoplaste. Plastes avec grains d'amidon.

Lead staining $(\times 3,600)$. A grain during rehydration.

Vacuoles are progressively developping from aleuron grains.

Globoids are rejected towards the tonoplast. Plastids with starch grain.

FIG. 4

Coloration au plomb $(\times 3600)$. Cytoplasme d'un grain bien hydraté.

La dégradation des globoides se poursuit. Les vacuoles sont maintenant bien développées.

Lead staining $(\times 3,600)$. Cytoplasm of a well hydrated grain.

Globoids are progressively reduced, and vacuoles are now well developped. 
Ces résultats rejoignent ceux de GiLissen (1977) sur pollen de Petunia hybrida, qui a montré que, par prétraitement en atmosphère saturée d'eau, on poııvait restaurer une germination normale de grains inaptes à germer immédiatement après stockage en atmosphère à $H R=0$ p. 100 . L'auteur relie cette différence de germination à une différence d'aptitude au gonflement initial des grains, elle même associée à une différence de porosité et d'épaisseur des parois du pollen et des membranes plasmiques selon l'atmosphère de stockage.

\subsection{Etude cytologique de la réhydratation}

L'observation en microscopie électronique de grains très déshydratés est rendue pratiquement impossible à cause des difficultés de fixation et d'inclusion. Nous n'avons cependant pas essayé la technique de OPIK (1980) qui, par séjour prolongé de l'échantillon en présence de vapeurs de tétroxyde d'osmium permet une fixation anhydre. Donc, sans aller jusqu'aux teneurs en eau très basses, nous avons pu noter une évolution de la structure de certains organites du cytoplasme, en particulier les mitochondries et les vacuoles, que l'on peut incontestablement attribuer aux effets de la réhydratation.

Dans les grains peu hydratés, les mitochondries apparaissent grandes, sphériques, claires et dépourvues de crêtes (fig. 1), aspect assez typique d'une mitochondrie non fonctionnelle. Au cours de la réhydratation, les crêtes se développent progressivement et les mitochondries prennent une forme plus allongée, caractéristique de mitochondries fonctionnelles (fig. 2).

De même, à un stade d'hydratation peu avancé, on distingue des structures identifiables à des grains d'aleurone (fig. 3). En cours de réhydratation, on note le développement progressif des vacuoles à partir de ces grains d'aleurone en voie de dégradation (fig. 4). Enfin, à ce stade, les grains d'amidon sont bien visibles dans les très nombreux plastes.

Cette évolution cytologique, en particulier au niveau des mitochondries et des vacuoles, est tout à fait analogue à celle qui est décrite par certains auteurs sur divers embryons mis en germination (Nougarede, 1963 a - 1963 b, sur Tropacolum majus; Hallam et al., 1972, sur Secale cereale; Vozzo, 1973, sur Quercus nigra).

\section{Conclusion}

Ce travail montre bien quiun grain de pollen n'est pas systématiquement apte à germer immédiatement, au moins dans les conditions d'une germination in vitro. Lors de la déshydratation rendue nécessaire pour sa conservation, le pollen perdrait, de manière réversible, l'aptitude à absorber très rapidement une grande quantité d'eau, phénomène qui se produit normalement au début de sa germination. On peut par contre réaliser un prétraitement du pollen en atmosphère humide, ce qui assure une première phase de réhydratation lente au cours de laquelle le métabolisme se réactive. Durant cette phase, la teneur en eau s'élève pour atteindre au bout de $16 \mathrm{~h}$. une valeur voisine de celle d'un pollen considéré au moment de sa déhiscence en conditions naturelles, et l'on constate parallèlement une évolution des vacuoles et du chondriome. Mais il est clair qu'une telle évolution ne pourrait être instantanée. Comme chez plusieurs autres espèces, 
(Duffield \& SNOW, 1941 : Visser, 1955; KInG, 1965 : Jensen, 1970 ; Gilissen, 1977 ; MuREN et al., 1979) on voit donc que pour du pollen conservé après déshydratation, la scule introduction de cette phase de réhydratation ménagée permet à certains lots de retrouver un taux élevé de germination. Cette constatation pourrait permettre d'améliorer les techniques de conservation en autorisant une déshydratation plus pousséc que celle qui est actuellement conseillée. On peut notamment penser aux techniques de lyophilisation rarement utilisées pour le pollen d'arbres forestiers. Il resterait enfin à déterminer si ce phénomène qui se manifeste in vitro, peut aussi se produire lors de pollinisations artificielles : une telle expérimentation est actucllement en colirs. Quand on sait limportance que pourrait prendre ce type de pollinisation dans les vergers à graines, on voit à quel point il y aurait lieu d’approfondir les études portant sur la conservation du pollen d'arbres forestiers et sur son utilisation.

Regu pour publication le 26 octobre 1982.

\section{Remerciements}

Nous tenons tout spécialement à remercier Mademoiselle L. Chesnoy, du Laboratoire de Cytologie et Biologie de la Reproduction, Université Paris VII, pour l'aide bienveillante qu'elle nous a apportée dans la partie cytologique de ce travail.

\section{Summary}

The influence of a rehydration phase before the in vitro germination of douglas-fir pollen (Pseudotsuga menziesii) after storage

The conditions for in vitro germination of douglas-fir pollen were precised. Then, special conditions for the use of dessicated pollen, after storage, were investigated. A slow hydration appeared to be a decisive phase for the early processes of pollen germination. A pretreatment for 16 hours at $26^{\circ} \mathrm{C}$, under $100 \mathrm{p}$. 100 relative humidity, was enough to allow more than 65 p. 100 pollen grains to germinate in a pollen lot germinating less than 13 p. 100 without prehydration. A cytological study revealed a strong evolution of the chondriom and vacuoles during this hydration phase.

\section{Références bibliographiques}

Allen G.S., Owens S.W., 1972. The life history of douglas-fir. Environment Canada, Forestry Service, BC-x-65, Ottawa, 139 p.

Barner H., Christiansen H., 1962. The formation of pollen, the pollination mechanism, and the determination of the most favourable time for controlled pollination in Pseudotsuga menziesii. Silvae Genet., 11, 89-102.

Bar-Shalom D., Mattsson O., 1976. Mode of hydration, an important factor in the germination of trinucleate pollen grain. Bot. Tidsokr., 71 (3-4), 245-251.

Brewbaker J.L., Kwack B.H., 1963. The essential role of calcium ion in pollen germination and pollen tube growth. Am. J. Bot., 50, 859-865.

Duffield J.W., SNow A.G., 1941. Pollen longevity of Pinus strobus and Pinus resinosa as controlled by humidity and temperature. Am. J. Bot., 28, 175-177.

GILISSEN L.J.W., 1977. The influence of relative humidity on the swelling of pollen grain in vitro. Planta, 137, 229-301. 
Glauert M.A., Glauert R.H., 1958. Araldite as an embedding medium for electron microscopy. J. biophys. biochem. Cytol., 4, 191-194.

Hallam N.D., Roberts B.E., Osborne D.J., 1972. Embryogenesis and germination in Rye (Secale cereale L.). II. - Biochemical and fine structural changes during germination. Planta, 105, 293-309.

JENSEN C.J., 1970. Some factors influencing survival of pollen on storage condition. Proc. I.U.F.R.O. Sect. 22, Working group on the sexual reproduction of forest trees, Varparanta, Finland, 28-5/5-6-1970, Pap. n“39, 18 p.

KING J.R., 1965. The storage of pollen-particulary by the freeze-drying method. Bull. Torrey bot. Club, 92, 270-287.

Muren R.C., Ching T.M., Ching K.K., 1979. Metabolic study of douglas-fir pollen germination in vitro. Physiol. Plant, 46, 287-292.

Nougarede A., 1963 a. Premières observations sur l'infrastructure et sur l'évolution des cellules des jeunes ébauches foliaires embryonnaires du Tropaeolum majus $\mathbf{L}$. : cytologie de la déshydratation de maturation. C.R. Acad. Sci. Paris, 257, 1335-1338.

Nougarede A., $1963 \mathrm{~b}$. Premières observations sur l'infrastructure et sur l'évolution des cellules des jeunes ébauches foliaires embryonnaires du Tropaeolum majus L : cytologie de l'hydratation germinative et des premières étapes de la germination. C.R. Acad. Sci. Paris, 257, 1495-1497.

OpIK H., 1980. The ultrastructure of coleoptile cells in dry rice (Oryza sativa L.) grains after anhydrous fixation with osmium tetroxyde vapour. New Phytol., 85, 521-529.

Simons D.H., Sfakiotakis E., Dilley D.R., 1972. Enhancement of in vitro pollen germination of lily with increased pre-inoculation humidity. Hort. Sci., 7 (6), 556-557.

Snyder E.B., Clausen K.E., 1974. Pollen handling. In seeds of woody plants in the United States, U.S.D.A. Agricultural Handbook, $\mathrm{n}^{\circ}$ 450, chap. IV, 75-97.

VISSER T., 1955. Germination and storage of pollen. Meded Landbouwhogesch., Wageningen, 55, $1-68$.

Vozzo J.A., 1973. Anatomic observations of dormant, stratified and germinated Quercus nigra embryos. Phytomorphology, 23, 245-255. 